\title{
Problems of Learning Arabic by Non-Arabic Speaking Children: Diagnosis and Treatment
}

\author{
Abdallah Hussein El-Omari \\ Department of English Language and Literature, Irbid University College, Al-Balqa' Applied University, Jordan \\ Hussein Mohammad Bataineh \\ Department of Arabic Language and Literature, Irbid University College, Al-Balqa' Applied University, Jordan
}

\begin{abstract}
The problem of Arabic learning difficulty is amongst the serious problems encountering the educationalists which would be attributed to many reasons. Therefore, this research is about the idea of detecting the real and minute reasons of Arabic learning problems by non-Arabic speaking children. Investigating and detecting these reasons are not the objective and substance of this research, but it is a reasonable method to diagnose the disease and set the proper linguistic cure prescription to extinguish the phenomenon of language weakness of these children. The research deals with linguistic treatment methods of this weakness at different phonetic, semantic, syntactic, morphological and lexical levels and areas. It also deals with solutions, suggestions, and recommendations which would take part in solving the problems of this serious problem.
\end{abstract}

Index Terms-Arabic, learning, problems, non-Arabic, speaking, children

\section{INTRODUCTION}

Arabic language issues have turned to be international, not only because it is amongst the eleven international languages, but also for other issues resulted from the current political and social conditions that the Arab world witnesses. Arabic issues have begun to diverse and expand as per the regional and international circumstances that the Arab world currently witnesses.

This inevitably has its consequences on most Arabic issues, sciences and affairs inside and outside the Arab world, as well as Arabic speakers and those who do not speak it. Nature of Arabic is not necessarily like that one which it enjoys in non Arabic speaking regions. Flourishing or declining of Arabic in the Arab world does not necessarily reflect its nature in non Arabic speaking countries. Handling language issues would not generally include its status outside its original environment. However, any cultural weakening inside the language nation will inevitably result in negligence of the language and ignoring its importance in society and generally in life.

\section{OBJECTIVES}

The research aims at setting an approved mechanism to teach Arabic to non-Arabic speaking people, besides making use of the latest linguistic, psychological and educational theories to improve the non-Arabic speaking children's performance and get rid of their language weakness. It also devotes proper and sufficient interest in teaching it to the non-Arabic speaking children, and to improve the output of advanced and extensive courses and educational programs in this regard, in addition to ensure the importance of childhood stage in language acquisition. The research moreover, aims to detect the aspects of similarity and difference amongst Arabic and non-Arabic speaking children regarding their ability to acquire language and problems of learning it.

\section{METHOD}

The research employs the descriptive approach, and uses the analytical, comparative, conclusive, deductive, explanatory, and representative methods besides resorting to mental references and scientific measurements. The research seeks objectivity and academic tendency as well as scientific credibility and trustful documentation. It tends to avoid the compositional style, arbitrary intolerance, and emotional inspiration which do not fit the scientific approach applied in rigorous scientific research and studies.

\section{DISCUSSION}

The problem will be investigated through two main approaches; reasons of language weakness of non-Arabic speaking children (NASC) and secondly treatment.

First - Reasons of language weakness of non-Arabic speaking children (NASC) 
There are factors and reasons which hinder the linguistic growth movement of NASC. These factors and reasons vary according to conditions, data and sources which can be categorized into general lists consisting of smaller particles and minor reasons attached to them. Therefore, it can be said that NASC's language weakness reasons are attributed to the following items:

- The individual:

It means here the child individually who represents the substance of language weakness phenomenon since this child is the language producer and articulator. Therefore, this child is the party of the most practice and effect to control the language process either strength or weakness. The child has age properties which control the mechanisms of dealing and approaching him / her as the mental, physical and psychological capabilities haven't been completed yet. Instead, the child undergoes many factors and circumstances which contribute to build his / her personality wellbeing. The focus here is on the child as an individual without investigating the family and social environment. In fact the child's mental, psychological and physical nature which is investigated away from the outer and surrounding effects. Consequently, the sub-reasons listed under the major one are frequent and distribute into a group of linguistic problems which directly cause the language weakness of the child. All these various reasons are categorized under one term which is called "language and communication disorder". This term has the following branches (Hawarneh, 2010):

First: Articulation disorder, consists of; omission, substitution, addition, and distortion.

Second: Voice disorder, consists of; monotonous, voice loudness, breathiness, and aphonia.

Third: Speech disorder, consists of; stuttering, blocking, and cluttering.

Fourth: Language disorder, consists of; language delay, aphasia, dysgraphia, dysnomia and apraxia, echolalia and agnosia, dyslexia, and language disability.

These features which hinder the individual's language growth process are, in fact, common with all children in the world and are not specific with the language speakers or others, though. They are actually scattered all over the world. Causes of these disorders are attributed to reasons, some of which are related to the child and others are related to the family and social environment. This research is excluded to those relate to the child's health, physical, psychological, and organic problems according to the following reasons:

First: Genetic reasons: They focus on the shortcomings which the child has since birth and carries from parents and grandparents. Burt (in Halabi, 2000) has found that $60 \%$ of the speech disorder cases he studied were affected by the genetic factor, and $32 \%$ of the 97 children's stuttering, murmuring and nuzzling cases of the study had a parent or a relative who has a similar case.

Second: Organic reasons: This is reflected by a birth problem to an organ of the child's articulation system such as tongue ligaments distortion, teeth, upper lip, jaws, and uvula defects, lower jaw joint inflection, and others. Studies indicate that articulation organs functional disorder with disagreement amongst them would be attributed to structural formation disorder. This would also be attributed to cerebral nerve, cortex, uvula, throat, nose, or ear damage. Distortion of teeth alignment, weak senses especially hearing, mental weakness and forcing the left-handed child to use the right hand are examples of organic reasons (Hawarneh, 2010).

Third: Psychological reasons: psychological factor has a prominent effect in children's language growth or weakness. However, weakness factors are not always attributed to the physical side, but there are signs of language weakness attributed to close or deep psychological motives, though. Emotional disorder is at the same time accompanied with speech disorder, and the problems a child encounters early in life makes the disappointment he / she suffers at the first speech try is the issue which puts him / her in a discouraging situation for consistent speech (ibid). Therefore, a child's state of deprivation and emotional hunger causes a state of stress and psychological anxiety and constant emotion which lead to speech disorder such as stuttering and blotting or complete refuse to talk because of the dissatisfying feeling which creates a reaction represented in psychological anxiety (Khleidi, 1997).

\section{- The Family}

Family is the place where children find warmth and loving environment to live in and eventually causes children's language to be affected especially by the mother who is the most important person in the process. That is why the child adopts ideas and behaviors of the mother through the close and intimate contact between the mother and her child. The effect asserts itself when comparing the language of the children at shelters and that of those having normal family life (Hawarneh, 2010).

The mother headed family atmosphere affects the aspects of strength and weakness of the child's language growth. Viewing the children with language intelligence and retaining big resource of vocabularies, structures, sentences and figurative linguistic images and styles, indicates that they were brought up in ideal and high class families. On the contrary, idle children suffering from language weaknesses live in a disordered, unstable, and tense atmosphere. Therefore, educated families encourage their children to practice language skills and learn more than one language besides their mother tongue. So, they proudly send their children to bilingual schools and enroll them in language learning courses.

An important issue in this regard is the family's religious culture. Muslim families usually care about the basic language of their religion and the Holy Koran. Therefore, families which do not care about their Islamic culture, their children usually suffer from language weakness leading to using poor vocabularies expressing their meanings and social relationships practiced in the family which entails more speech linguistic supplies in the culturally poor environment. 
This leaves the child of such environments repeating the same limited vocabularies and unable to acquire new utterances to make speeches meaningful (ibid).

\section{- The Society}

Society is the second circle surrounding the child after that of the family. It is the close and supplementing boundary of the family. Its effect could be direct or indirect on the child through affecting the family. It is the magnetic field which the child orbits in outside the house. Therefore, the society could be a direct or indirect reason of the child's language weakness. The non-Arabic speaking society being interested in a second language such as Arabic entails the family's care about this which reflects on the non-Arabic speaking child. On the other hand, the less interest a nonArabic speaking society gives to learning Arabic means that the family lacks interest in the issue leading to the child's language weakness. Sometimes, a society lacking interest in its mother tongue is seen as a sufficient reason for being less considerate about other languages. The society's educational situation often affects the child's language growth, especially when the society is more interested in providing necessary life requirements of living, accommodation and jobs. Language learning becomes a secondary issue including learning a second one.

Other social issues which would cause language weakness, the religious attitude of the society could be mentioned here. Non-Arabic speaking Islamic societies such as Turkey and Malaysia pay a great attention to learning Arabic which might be more than that which they give to their mother tongues because of the sacredness and solemnity they have for Arabic being the language of the Koran and the religion of Islam. This would certainly affect the children's language growth of these societies.

\section{- International policies}

National policies of countries play a great role in the linguistic issues. This is reflected through the strong diplomatic relations among countries and peoples. A country taking the decision to use a second language for instructing is sometimes politically motivated. The second language might have no linguistic, scientific, or historical relationship with the mother tongue, and both of the two languages belong to different language families such as French in some Arab countries. This is usually affected and directed by the general policies and linguistic planning of these countries.

Arabic for example in non-Arabic speaking countries suffers from the poor attention given to it which is attributed to many considerations. There are very few centers for teaching Arabic to non-Arabic speaking communities, and if they exist they employ poor methods and use traditional techniques. Teachers with poor skills, who lack proper command of Arabic, inappropriate selection of textbooks and syllabuses and scarcity of programs and activities which would attract the non-Arabic speaking children, all these end up in poor performance. Moreover, Arab media especially TV channels do not properly care about the issue by not dedicating special channels addressing these non-Arabic speaking communities, or at least produce Arabic language teaching programs and serials in classical Arabic for its expected positive effect on the intended children as did foreign Western programs on Arab children. This technique might be used to help children get rid of their language weakness.

\section{LANGUAGE WEAKNESS TREATMENT}

Sartawi (2001) set a bunch of insightful questions which aimed at establishing a children language problems and weaknesses treatment process:

- What are the sounds which the individual makes mistakes at?

- How many wrong sounds are there?

- What is the type of these mistakes; is it omission, substitution, addition, or distortion?

- Where is the position of the mistakes; is it at the beginning, middle, or end of the word?

- What are the conditions of repeating the mistakes and being so many, and are they so only in fast talks or under all talk conditions?

- How do the patients' mistakes appear?

- What is the reason of the individuals' speech difficulties?

Are they attributed to recognition problems, recalling abilities, strength of memory, nerve ability, speech type competencies, or the motive and incentive to produce proper and correct speech (Sartawi, 2001)?

Sartawi's (2001) view is worth consideration for the extent of competence of the speech models which is an important point specifically for the NASC. Here a bunch of central questions could be asked which would contribute to highlighting this point. What is the nature of these models, who are to prepare them, and to what extent are they capable of covering the linguistic phenomenon?

Preparing linguistic models for the NASC is inevitably different from the models designed for the Arabic speaking children because of the following reasons:

First: Lessons provided to NASC are usually made easier than those of the Arabic speaking. Set the linguistic material for the children so that it meets their conditions and capabilities but at the same time, not to be too easy. It is preferred if these models are prepared by non Arabic speaking linguists with the participation of Arab linguists as they are aware of the problems of language learning.

Second: The cognitive and mental content of the linguistic material: 
Children should be put in an Arab atmosphere, i.e. to live the Arab environment with all its traditions and living conditions and styles. This entails choosing the names of people and places, lodgings, edibles, drinkables and clothes from the Arab environment. This means commitment to the Arab names and terms in the lessons.

Third: Teaching techniques:

This includes interest in the form of the study material such as abundant pictures, colors, graphs, line font, print and color. It also includes full editing of texts by adding all writing signs and punctuation marks, as well as quality of printing and paper used. Classes to be given in model classrooms including necessary preparations and teaching process requirements, audio and video.

Fourth: People in charge of the teaching process:

To treat language weakness of Arabic speaking children or NASC, competent teachers of high experience and excellent skills should inevitably be selected, as it is not any teacher is capable to take the responsibility of such a task. Such a teacher chosen to take the responsibility of teaching language or treating the language weakness and should obtain the competences of a successful teacher. These include acquaintance of the educational side and child psychology, i.e. to be educationally well qualified to take both processes of language teaching and treating the language weakness besides obtaining necessary skills to perform tasks for their influence on work accuracy, swiftness, saving time and effort, and expanse. Moreover, these skills provide educationists with power, self confidence, and perfection. Teachers should also embrace high educational ethics since they are examples for their students inside and outside the educational bodies which would reflect on the students' behavior and ultimately accept and embrace the language of their examples. It is very much preferable if those in charge of the teaching process of NASC among those who have learnt Arabic from native speakers directly or studied Arabic in Arab universities in Arabic speaking countries. This would enable the teachers to truly represent pure Arabic from its sources and transfer it with all its features, phones, tunes and intonations to the NASC.

Fifth: Comprehension of linguistic models of language syntax:

This is performed by comprehending phonetic, syntactic, morphological, lexical, and semantic models so that all linguistic levels would be covered. Models and examples are equally and evenly distributed so that focus does not fall on a linguistic level rather than another which would prevent slouch in a linguistic side or shrink in another.

Sixth: Analysis of children literature educational content:

Nothing would be so firm in children's mentality as songs, merry expressive poems, linguistic modules built on rhythmic phones, balance between expressions, and nice divisions. Children literature is a high kind of art, and poetry in specific is the nearest to children's psyche and the most effective on it. It is the food of soul and amuse of the heart and eye. It discloses the meaning of things which the heart and eye resort to. It is the sixth sense lying deep in one's psyche. It is music, poetry, harmony, rhythm and rhymed speech which children adore (Kan'aan, 1995).

Starting from this idea and the effect of children literature in language teaching and treating its problem and defects, the following should be done:

- Giving great care to children literature at learning Arabic by the NASC.

- Well selecting of literary works by Arab poets which meet all age stages of childhood.

- Children memorizing merry and nice songs of appropriate tones and rhythms.

- Poems should include authentic, social, practical, cognitive and human Arab values.

- Children literature should be ordered in light of Islamic teachings since Arabic is the vehicle of Islam.

Therefore, analyzing the educational content of the songs is inevitable besides the outer appearance, rhythm and music. Content of the educational literature besides its ethical role would support children's linguistic skills and foster vocabularies. It would also increase children's lexical reservoir and get them be more efficient users of expression styles.

Content analysis is a new technique of scientific research which widely emerged in all walks of life. This term, "content analysis" is a method of studying communication topics in a methodological, objective and quantity style to measure variables and appoint the degree of germination or repetition of a certain communication phenomenon such as calling, methods, content change and difficulty degree of a text. It is a method of observation and measurement together. It is observing people's behavior through analyzing their linguistic production (ibid, p. 15).

Therefore, texts and works are not irregularly selected, and they are not only chosen according to the impression they have on the teachers' psyches. It should also comply to the content analysis technique, so that their selection would be structured on scientific bases not on self subjective motives and self impressions governed by individual interests and tastes. Content analysis is rooted back to the eighteenth century when quantity was taken as the standard of analyzing recitations and religious preaching by counting the expressive words in them (Rosengren, 1981).

Therefore, those in charge of preparing textbooks and selecting texts and songs have to be trained to use the skill of content analysis of the courses they perform. There are some books about this field which should be read and understood. One of the famous books for instance is Pearlson's 'Content Analysis in Media Research' which was published in 1952, and Holsny's 'Content Analysis in Social and Human Studies' published in 1969, and Kerbendrof's 'Content Analysis, Stylistic Introduction' published in 1980.

It should be known that content analysis is generally about analyzing linguistic and semantic features of communication symbols and identifying recurrence and existence of these features with a high degree of sharp strict 
control or limiting the quantity values of these repetitions. In fact, this is the view of the two researchers Letts and Paul in 1942 (in Hussein, 1983).

Content analyzers' findings are distinguished with objectivity, reliability, consistency, quantity, quality, publicity (circularity), analyzing and applying on all issues and cooperation with other methods and modern techniques. These are among the most prominent content analysis features, especially nowadays with the mass use and availability of the computer which facilitate its application (Kerlinger, 1973).

Seventh; Motivation:

NASC's language acquisition differs from their mother tongue since the latter is the first language they are exposed to and hear and speak before Arabic - which is second. This makes its acquisition difficult and more complicated. However, acquiring the mother tongue and a second language is subject to common denominators. For instance, Skinner's concept of motive and response and all that accompanies it are active standards in acquiring the mother tongue and a second language. Skinner thinks that language acquisition takes place in a social medium by the motive and response. He inserts the performance behavior concept and finds that the vocative behavior is like the performance behavior which can be supported (Skinner, 1957). Summing up Skinner's belief, it is about support, reward, and enhancement in children's language acquisition, since responses that do not meet support and reward burnout (Assayed, 2004). Relying on this point, a question arises: what are the proper methods to encourage and motivate NASC to acquire it, and what is its grade according to its importance?

Means of motivation: There is a group of successful means to motivate NASC to learn Arabic which effectively takes part in Arabic learning problems treatment of those children. The most prominent of which are:

- Television

It is the means which highly attracts children's interest by following and watching it. It the primary means of controlling their interests and mentalities amongst other media means. It is a double edged weapon, as it may plant positive or negative values in children's personality. Therefore, positively speaking it has several benefits of treating children's problems of Arabic language learning in general and NASC in specific through the informative content it carries especially in its linguistic side. It is the age of technology and image that the paper culture is being threatened to deteriorate for the electronic culture and television. Television is much faster and stronger than conventional communication means (Fifi, 2010). Serials and children programs in classical Arabic are encouraged on television besides screening simple educational programs and songs for children.

- Competitions and awards

Competitions and awards might be the most important methods to motivate NASC to learn Arabic, and concentration should be given by concerned educational and linguistic authorities as effective methods to encourage children to overcome Arabic learning difficulties. Moreover, children would skillfully learn articulating and understanding Arabic by using these methods. This requires the existence of a supporting financial authority. Supporting such competitions and awards might not be that costing, since a reasonable small amount of money would be enough.

Therefore, programs of teaching Arabic to NASC should be funded including the centers of teaching it as well as setting advanced and intensive courses and rewarding all participants - those who are distinguished and equally those who only passed, even though the awards are moral or symbolic. The effect awards leave on learners is the most important. It would be rather better if the awards were language related, such as Arabic books, magazines, stories, and dictionaries - paper or speaking electronic. It is also preferable if the NASC who suffer from language learning be taken to Arabic speaking countries as a kind of reward. There they would mix, converse realize proper styles of speaking the language naturally. They would visit schools, attend classes, do sightseeing, wander in the streets, read shop signs and merge in a true Arabic atmosphere. Ancient Arabs did so in the pre-Islamic era by sending their children to stay with the original Arab Bedouins to learn pure Arabic.

- Language games

Language games are interesting children attraction as they meet their natural tendency to play. They are intended and designed for competition among the opponents for an educational learning aim. They are amongst the best helping means of practicing language as they depend on interactive collaborative learning, where each participant has a role to play. They are also among the approved means of Arabic teaching to all learners and non-Arabic speakers in specific. Their importance stems from separating teaching away from rigid and artificial learning. They enrich teaching and learning and encourage children to use the language and keeps classes or learning sessions away from routine and boredom, and help children understand many of linguistic issues and free them from fear and shyness. They depend on giving:

First: Live games which children themselves perform or be part of it. Amongst its typical examples is ordering letters of the alphabet to form words, or cards to form sentences, or match words with pictures, or fill in missing letters or words. These forms are not for fun only, but for teaching and learning.

Second: Electronic games which are more common in the age of globalization, digital means and figures. They are more attractive for children than previous games, but they are more harmful and dangerous that they should be avoided as they affect sight and the skeleton and cause other harms. These types of games affect personality as they are usually performed by one player, so it should be massively performed by using display devices. Language games should include a sense of competitiveness which conveys suspense and language enrichment to encourage children to interact 
as there are rewards and awards for the winners. Main aims of these language games in the teaching process might be briefed in teaching thinking and teaching children Arabic conversation through continuous talk which helps children articulate properly. They also aim at enriching children's language output as well as combining learning and fun and fostering language skills and experiences of learners as they are linked with unforgettable fun and games.

\section{REFERENCES}

[1] Assayed, Mahmoud. (2004). Methods of Teaching Arabic, Faculty of Education, Damascus University, Syria, unpublished PhD thesis.

[2] Fifi, Abdullah bin Ahmad. (2010). Arab Culture in Light of Modern Communication Means, Al-Arabi Magazine, , Al-Arabi Book, No. 82, $1^{\text {st }}$ Ed. Published by the Ministry of Information, Kuwait.

[3] Halabi, Muwafaq. (2000). Psychological disturbance of Children and Teenagers, $2^{\text {nd }}$ Ed. Published by Arresalah Establishment.

[4] Hawarneh, Mu'ammar Nawaf. (2010). Children language and communication disturbance "phenomenon and treatment", General Syrian Book Corporation Publishes, Ministry of Culture, Damascus

[5] Hussein, Sameer Mohammed. (1983). Content Analysis, 'Aalam Alkutub, $1^{\text {st }}$ Ed., Cairo.

[6] Kan'aan, Ahmed Ali. (1995). Children Poetry in Syria "Study of Educational Content Analysis", Publishes of Arab Writers Union, Damascus.

[7] Kerlinger, Fred N. (1973). Foundation of Behavioral Research, Edel Holtrine Hart and Wistan Inc., New York University, p. 527.

[8] Khleidi, Abdulmajid \& Wahbi, Kamal Hassan. (1997). Psychological and Mental Diseases and Behavioral Disturbances of Children, $1^{\text {st }}$ Ed. Publisher: Dar Alfikr Alarabi, Beirut.

[9] Rosengren, Karl Erik. (1981). Advances in Content Analysis, printed in USA, p. 9.

[10] Sartawi, Abdulaziz Mustafa. (2001). Language and Talking Disturbance, $1^{\text {st }}$ Ed. Published by Academy of Special Education, Riyadh, KSA.

[11] Skinner B. F. (1957). Verbal Behavior, N. Y. Appleton Century Crofts.

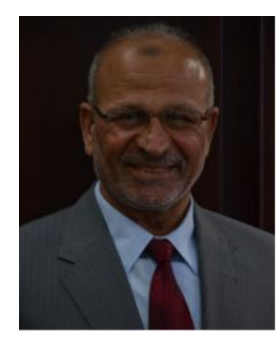

and International.
Abdallah H. El-Omari, born in Jordan, 1953. GSC, 1972. BA in English Language and Literature, Beirut Arab Univ., Lebanon, 1976. MA in Translation, Yarmouk Univ, Jordan, 1991. Short Course in Advanced English and American Studies, School of Advanced International Studies SAIS, Washington DC, of Jones Hopkinz University Baltimour, USA 1989. PhD in Methods of Teaching English, Malaysia National Univ, UKM, Bangi, Malaysia, 2002. (Strategies and methods of language teaching)

He worked as a Teacher of English in Libya, Jordan, and Saudi Arabia. Translator and Interpreter (English/Arabic, Vice versa) in Jordan and Saudi Arabia. Currently: University Instructor of English Language and Literature and Associate Prof. of Curriculum and Methods of Teaching English at Balqaa' Applied University, Jordan. Several Publications of papers in Journals and Conference proceedings, Local

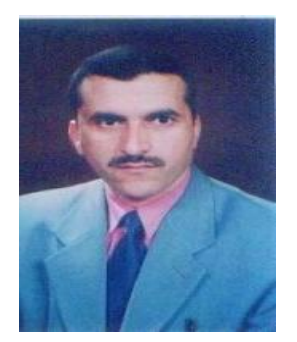

Hussein M. Bataineh, born in Jordan, 1966. GSC, Jordan, 1984. BA in Arabic language and Literature, Yarmouk Univ. Jordan, 1988. MA in Arabic language and syntax, Yarmouk Univ. 1998. PhD, Arabic language and syntax, Nelein Univ. The Sudan, 2001. Teacher of Arabic, and educational supervisor at the Ministry of Education, Jordan. Currently: University instructor and Associate Prof. of Arabic language and literature at Balqaa' Applied University, Jordan. Published several papers and articles in Journals and conference proceedings, local and international. 